Open Access

\title{
Developing clinical decision tools to implement chronic disease prevention and screening in primary care: the BETTER 2 program (building on existing tools to improve chronic disease prevention and screening in primary care)
}

Donna Patricia Manca ${ }^{1,2^{*}}$, Denise Campbell-Scherer ${ }^{1,2+}$, Kris Aubrey-Bassler $^{3 \dagger}$, Kami Kandola $^{4 \dagger}$, Carolina Aguilar ${ }^{1 \dagger}$, Julia Baxter ${ }^{5 \dagger}$, Christopher Meaney ${ }^{5 \dagger}$, Ginetta Salvalaggio ${ }^{1 \dagger}$, June C. Carroll ${ }^{5 \dagger}$, Vee Faria ${ }^{4 \dagger}$, Candace Nykiforuk ${ }^{6 \dagger}$, Eva Grunfeld ${ }^{5,7+}$ and the original BETTER Trial Investigators and Clinical Working Group

\footnotetext{
Abstract

Background: The Building on Existing Tools to Improve Chronic Disease Prevention and Screening in Family Practice (BETTER) trial demonstrated the effectiveness of an approach to chronic disease prevention and screening (CDPS) through a new skilled role of a 'prevention practitioner'(PP). The PP has appointments with patients 40-65 years of age that focus on primary prevention activities and screening of cancer (breast, colorectal, cervical), diabetes and cardiovascular disease and associated lifestyle factors. There are numerous and occasionally conflicting evidence-based guidelines for CDPS, and the majority of these guidelines are focused on specific diseases or conditions; however, primary care providers often attend to patients with multiple conditions. To ensure that high-level evidence guidelines were used, existing clinical practice guidelines and tools were reviewed and integrated into blended BETTER tool kits. Building on the results of the BETTER trial, the BETTER tools were updated for implementation of the BETTER 2 program into participating urban, rural and remote communities across Canada.

Methods: A clinical working group consisting of PPs, clinicians and researchers with support from the Centre for Effective Practice reviewed the literature to update, revise and adapt the integrated evidence algorithms and tool kits used in the BETTER trial. These resources are nuanced, based on individual patient risk, values and preferences and are designed to facilitate decision-making between providers across the target diseases and lifestyle factors included in the BETTER 2 program. Using the updated BETTER 2 toolkit, clinicians 1) determine which CDPS actions patients are eligible to receive and 2) develop individualized 'prevention prescriptions' with patients through shared decision-making and motivational interviewing.

(Continued on next page)
}

\footnotetext{
* Correspondence: dpmanca@ualberta.ca

${ }^{\dagger}$ Equal contributors

'Department of Family Medicine, University of Alberta, 6-10 University

Terrace, Edmonton, Alberta T6G 2T4, Canada

${ }^{2}$ Covenant Health, Grey Nuns Community Hospital, 1100 Youville Drive

Northwest, Edmonton, Alberta T6L 5X8, Canada

Full list of author information is available at the end of the article
} 


\begin{abstract}
(Continued from previous page)
Results: The tools identify the patients' risks and eligible primary CDPS activities: the patient survey captures the patient's health history; the prevention visit form and integrated CDPS care map identify eligible CDPS activities and facilitate decisions when certain conditions are met; and the 'bubble diagram' and 'prevention prescription' promote shared decision-making.

Conclusion: The integrated clinical decision-making tools of BETTER 2 provide resources for clinicians and policymakers that address patients' complex care needs beyond single disease approaches and can be adapted to facilitate CDPS in the urban, rural and remote clinical setting.
\end{abstract}

Trial registration: The registration number of the original RCT BETTER trial was ISRCTN07170460.

\section{Background \\ Context}

The prevalence of chronic disease is steadily increasing $[1,2]$, and primary care is an ideal setting for chronic disease prevention and screening (CDPS) activities [3-5]. Regrettably, evidence-based tools and strategies for CDPS are inconsistently applied in the primary care setting, in part due to the numerous and sometimes conflicting recommendations and guidelines [6, 7]. Since $45 \%$ of people have one or more chronic disease [8], primary care providers need effective strategies that address multiple conditions. However, guidelines are focused on specific conditions or risk factors $[7,9]$, which makes it difficult for clinicians to address patients' unique risk profiles. Thus, a comprehensive evidence-based approach to CDPS has been 'lost in translation' [10] and there is a need to engage end-users including clinicians, researchers and policymakers in a collaborative process [10] to address this knowledge to action gap. Furthermore, with the competing demands on primary care providers there is little time to address CDPS [11, 12]; hence, a new approach that bridges the evidence to practice gap in primary care CDPS is needed.

The Building on Existing Tools to Improve Chronic Disease Prevention and Screening in Family Practice (BETTER) trial was a pragmatic two-way factorial cluster randomized controlled trial conducted in urban primary care team practices in Alberta and Ontario, Canada [12]. Patients aged 40-65 were invited to participate in the trial and stratified into groups: 1) general medical patients and 2) patients with moderate mental illness. The BETTER tool kit and training provided the 'prevention practitioners' (PP) in the two urban settings with the necessary tools and resources to evaluate patients for multiple risks. The tools were aimed to prevent multiple chronic conditions through a process of shared decisionmaking, which provided the patient with an individualized 'prevention prescription' that included actionable CDPS goals (see Fig. 1). The PPs in the BETTER trial were clinicians (licensed practical nurse, nurse, dietician, nurse practitioner) who worked in the multidisciplinary primary care clinics to develop a comprehensive approach to evidence-based CDPS within the practice setting $[7,12,13]$.

To bridge the gap between knowledge and practice, a BETTER trial clinical working group (CWG) was formed with end-users that included PPs, clinicians and researchers with support from the Centre for Effective Practice [7]. The CWG identified and harmonized highquality clinical practice guidelines and tools for primary CDPS in adults 40-65 years of age [7, 12], creating the BETTER tool kit that was implemented in the BETTER trial. This extensive review also defined the scope of the BETTER trial; the chronic diseases with the best evidence for primary prevention and screening were identified and incorporated into the comprehensive approach to CDPS. The target conditions included in the BETTER trial were cancer (breast, cervical, and colorectal), cardiovascular disease, diabetes and their associated lifestyle factors (smoking, alcohol consumption, diet/nutrition and physical activity). The BETTER trial demonstrated the effectiveness of a shared decision-making approach to CDPS that improved the implementation of clinically important CDPS activities through the new skilled role of a 'prevention practitioner' in both patient strata [12].

Once the BETTER trial demonstrated that a PP could improve the implementation of clinically important CDPS actions in a cost-effective manner [12], further funding was obtained to broaden the reach to other jurisdictions including urban, rural and remote communities in Canada and deepen the impact of the intervention through the Building on Existing Tools to Improve Chronic Disease Prevention and Screening in Primary Care (BETTER 2) program [14]. To achieve this goal and bridge the evidence to practice gap, revisions to the BETTER trial kit were required in order to update the evidence and adapt the tools into a format that could be used in diverse primary care settings including rural and remote settings and with aboriginal populations. This process was, in part, informed by feedback received from the participating clinicians and patients in the BETTER trial as it indicated that some of the items could be modified or removed, while others, such as the family history, could be better formatted to improve data capture. 


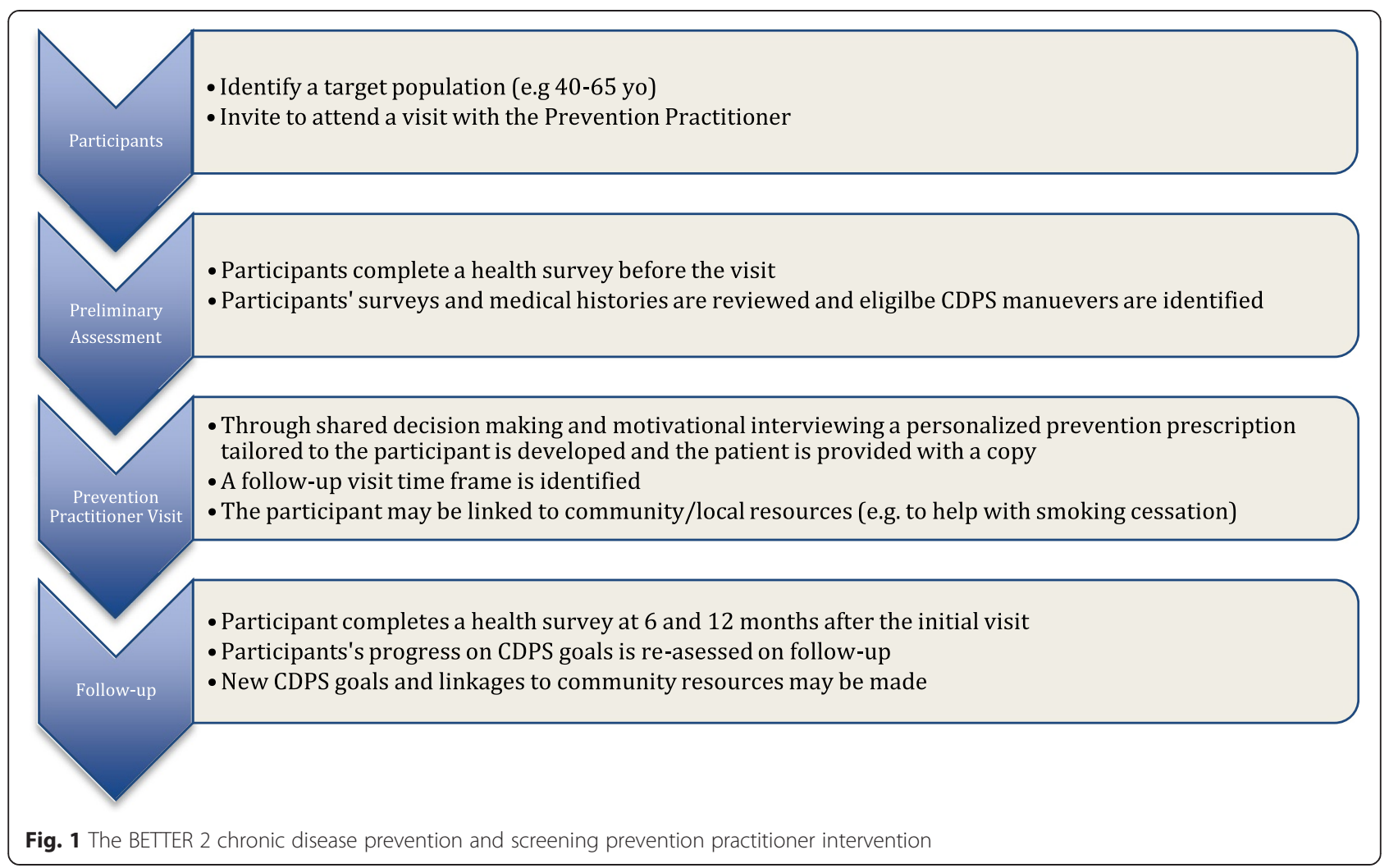

We describe here the process of integrated knowledge translation [15-18] that involved engaging end-users from the various practice settings (including clinicians and policymakers) with researchers as equal partners in this knowledge synthesis and the development of the resulting BETTER 2 tools.

\section{Purpose}

The purpose of this paper is to describe 1) the integrated process used to adapt and refine the BETTER trial tools for chronic disease prevention and screening (CDPS) by the BETTER 2 program and 2) the resultant tools that were then implemented into various urban, rural, remote and aboriginal primary care settings by the BETTER 2 program.

\section{Methods/design}

The BETTER trial CWG identified and reviewed highquality clinical practice guidelines and harmonized them to standardize the recommendations for implementation into the BETTER trial [7]. The CWG considered strong evidence that was linked to a target or health outcome [7] and created the knowledge products for the PP intervention that were used in the trial $[7,12]$. The knowledge products were developed from November 2009 to March 2010 through a structured approach to evidence integration [7] involving a knowledge to action cycle
(Fig. 2) [7, 10]. This involved engaging the researchers with end-users and policymakers in a process that included knowledge synthesis and harmonization through a structured evidence review process, and then testing and applying the tools in the various practice settings through an iterative plan-do-study-act (PDSA) process. Findings from the local PDSA activities were then integrated into the tools.

The BETTER 2 CWG was convened in November 2012; this group included end-users, clinicians (family physicians, registered nurses, nurse practitioners), policymakers from the new jurisdictions (Northwest Territories, Newfoundland and Labrador) and researchers tasked with reviewing and updating the high-quality recommendations for primary prevention of chronic conditions in patients 40-65 years of age. The chronic conditions included cardiovascular disease, diabetes and breast, colorectal, lung and cervical cancer, as well as the associated lifestyle risk factors (e.g. tobacco use, alcohol overuse, poor diet and physical inactivity) [7, 12]. A targeted search using the process described in our previous publication [7] was conducted to identify new resources meeting any of the following criteria:

1. Date of publication subsequent to 2009;

2. Addressing a gap or special population not considered in the original BETTER trial search; 


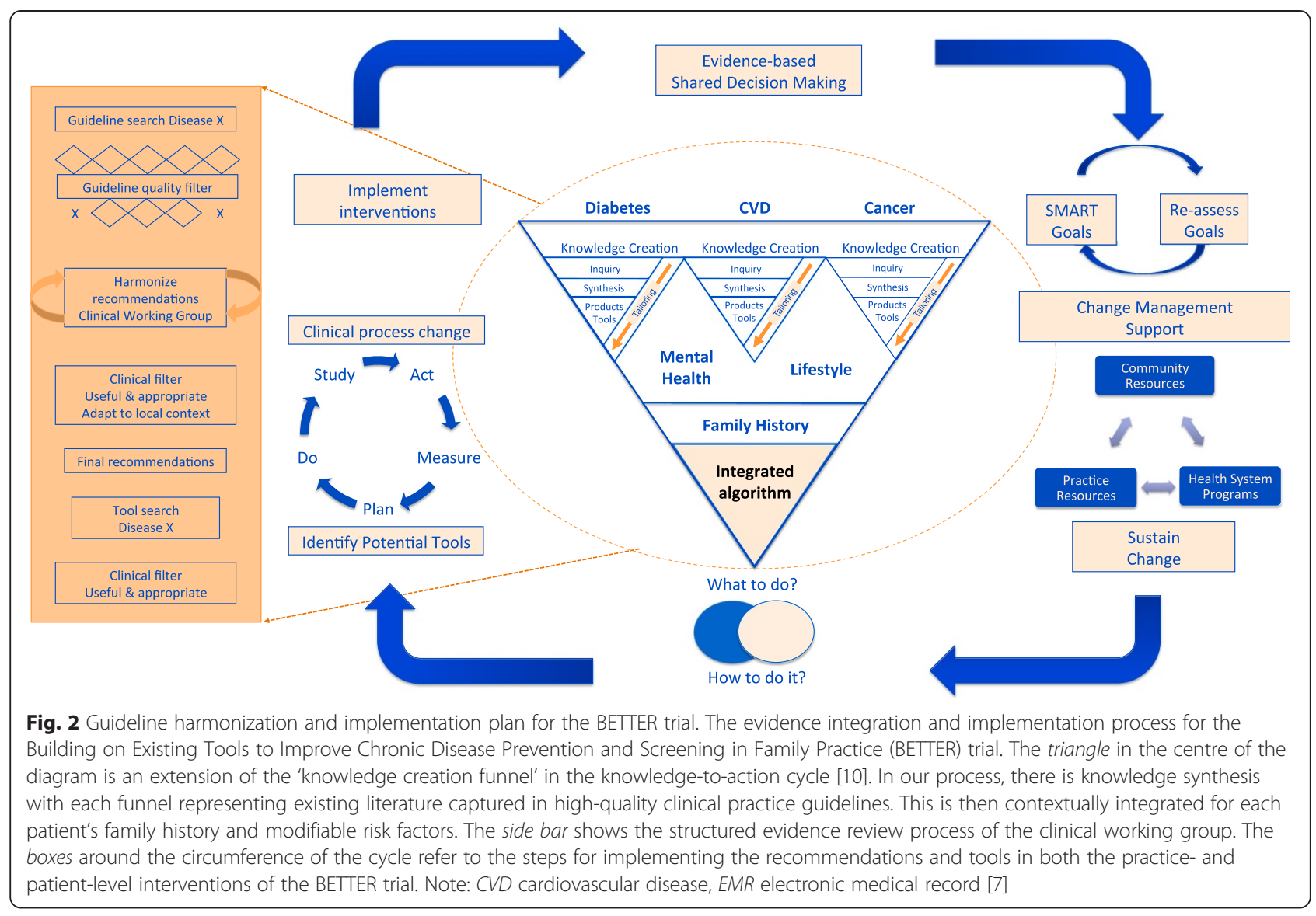

3. Interventions strongly recommended for application in practice;

4. Recommendations for patients at higher risk due to family history;

5. New resources identified through scoping reviews of provincial and territorial recommendations.

The CWG was divided into teams focusing on the following conditions: breast cancer, cervical cancer, colorectal cancer, skin cancer, lung cancer, cardiovascular disease, diabetes, alcohol, mental health, lifestyle (tobacco, alcohol, nutrition and physical activity), obesity (waist circumference, BMI) and family history.

Scoping reviews of provincial and territorial recommendations were also conducted to assist with further tailoring of the tools to comply with the approaches to CDPS in participating Canadian provincial or territorial jurisdictions (Alberta, Ontario, Northwest Territories, Newfoundland and Labrador). For example, a decision was made to reduce the consumption thresholds recommended in Canada's low-risk alcohol drinking guidelines [19] as the Northwest Territories was concerned with the high rates of colorectal cancer in their jurisdiction concomitant with heavy drinking [20] and the potential for increased cancer risk in those exceeding the alcohol levels recommended by the Canadian Cancer Society $[21,22]$. The CWG concluded that the Canadian guidelines were more focused on the risk of developing an alcohol use disorder and therefore did not adequately inform individuals about lower alcohol consumption levels to reduce the risk for chronic disease such as cancer. The tools were adapted to focus on informing Canadians about safer levels of alcohol consumption to prevent chronic disease, an approach that has since been recommended by the Canadian Cancer Society [22].

The members of the BETTER 2 CWG individual topic teams met, independently reviewed and critiqued the new information identified and presented their assessments to the CWG for review by the entire group in November 2012, December 2012 and January 2013. To address gaps and build on the previous work, a greater emphasis was placed on tools that would facilitate family history assessment, address local disease and risk factor prevalence and help harness local resources. Following this, the BETTER tools were updated, reviewed, edited and tested by various members of the BETTER 2 CWG to determine if they were useful and appropriate in the various clinical settings. The review and BETTER 2 tool kit refinement was completed in January 2013 after which training sessions were held with CWG members 
and PPs to implement the updated tools into the various practice settings.

\section{Results}

As a result of the comprehensive work of the BETTER 2 CWG, the tools were updated and adapted to address CDPS in the various urban, rural, remote and aboriginal contexts. The following tools were refined for inclusion in the BETTER 2 tool kit: a patient health survey (Additional files 1 and 2), a CDPS care map (Additional file 3), a prevention visit form (Additional file 4), the bubble diagrams (Additional file 5) and the prevention prescription with goals (Additional file 6). The tool kit can be accessed on the BETTER website [23]. This toolkit was the foundation for the comprehensive approach to CDPS implemented in participating jurisdictions and was further customized for each practice setting through the identification of local, regional and national resources that could be harnessed to support patients' CDPS care plans and lifestyle change goals.

\section{Using the BETTER tools}

The patient survey (Additional files 1 and 2) and prevention visit form (Additional file 4) capture the patient information and characteristics needed to make CDPS recommendations. The CDPS care map (Additional file 3), informed by the aforementioned data collection instruments, is a clinical decision aid that helps the clinician determine which CDPS recommendations the patient is eligible to receive when certain criteria a met, including when to refer the patient back to their primary care provider. The bubble diagrams (Additional file 5) are also instructive to both the clinician and patient as to the CDPS activities a patient is eligible to receive and can be used to facilitate agenda setting with the patient. The prevention prescription (Additional file 6) is a document intended for the patients to take with them when they leave the visit to help inform them of their prevention and screening status and guide patients on when, where and how they will go about improving deficient CDPS recommendations. Through a shared decision-making process between the clinician and patient, patients also set specific, measurable, attainable, realistic, timely (SMART) goals for their health (Additional file 6), providing the patient and clinician with a personalized plan geared toward enabling patients to achieve their CDPS goals.

\section{Patient survey}

\section{The BETTER survey}

The BETTER patient health survey (Additional file 1) provides primary care providers with a tool that captures the comprehensive patient information required to facilitate CDPS and monitor progress including the important behavioural, environmental and familial risk factors.
This tool comprises validated instruments (Additional file 2) that can gather detailed information relevant to CDPS including chronic conditions, previous cancer screening activities, lifestyle information and risks, perception of general health and depression, family history of certain medical conditions, food security and demographic information (age, gender, ethnicity, education, marital status, income). Much of this information is not routinely collected or available in the medical record; yet, this information is required for a clinician to determine the CDPS actions an individual patient should focus on.

The BETTER trial survey was refined for use in BETTER 2 based partly on feedback received indicating that the tool could be streamlined and reformatted to improve data capture and usability. The original patient health survey was lengthy, consisting of 88 items [12], and included an assessment of physical activity using activity recall [24] and a dietary assessment derived from the MEDFICTS, a dietary instrument with a focus primarily on fat intake $[25,26]$. After review by the BETTER 2 CWG, it was determined that employment of other validated tools to assess diet and exercise, which had been developed for use in primary care, could improve the ability to identify those patients who would benefit from a brief intervention and track changes over time.

Improvements to the original survey were made to better capture physical activity assessments including use of the general practice physical activity questionnaire (GPPAQ) [27] to determine the patients' level of activity in addition to the patients' self-reported number of minutes spent on exercise weekly to determine if patients are achieving a CDPS target of $\geq 150 \mathrm{~min}$ per week of moderate exercise [28-32]. The GPPAQ is a reliable and validated tool recommended for the assessment of physical activity in general practice that is supported by the United Kingdom National Institute for Health and Care Excellence (NICE) [27]. This tool assesses the respondent's level of physical activity both in and outside of work and can be used by clinicians to inform when an intervention to increase physical activity would be beneficial as well as to track a patient's progress over time.

A validated tool for dietary assessment and intervention in the clinical setting, Starting the Conversation [33], was added to provide the clinician with insight into patients' eating behaviours and information on how patients could improve their diet (e.g. increase fruit and vegetables, decrease sweetened beverages, decrease unhealthy snacks).

Alcohol consumption is now captured quantitatively to determine if patients are drinking within healthy alcohol consumption guidelines according to the National Institute on Alcohol Abuse and Alcoholism's overview of alcohol consumption for low-risk drinking [34]. Alcohol use disorders are also screened for using the 
validated abbreviated form of the alcohol use disorders identification test, the AUDIT-C [35-38]. The updated tools provide clinicians with an approach that educate patients about healthy alcohol consumption as opposed to only screening for abuse.

The health survey was reduced from 88 to 69 items to more efficiently capture information on the important modifiable lifestyle risk factors (smoking, alcohol consumption, diet/nutrition and physical activity) and includes assessments of the patient's readiness to change, gathering the information required to address these risk factors $[39,40]$. The health survey can take up to $30 \mathrm{~min}$ to complete. It may be completed before the patient's prevention visit either independently by the patient or administered by a health care professional when deemed appropriate (e.g. literacy, language).

\section{Prevention visit form}

The prevention visit form (Additional file 4) is a clinical tool that captures and structures the information obtained from the patient's survey and medical chart required to identify which prevention activities each patient is (or is not) eligible to receive. Typically, the clinician will partially complete this form before the patient visit to identify the CDPS activities eligible for discussion. Further information is collected at the time of the prevention visit including a limited physical assessment of the patient to obtain weight, height, waist circumference and blood pressure. Before the patient visit, the clinician can enter the patient's individual CDPS information on a blank version of the bubble diagram (Additional file 5) and the first page of the prevention prescription (Additional file 6).

\section{CDPS care maps}

The CDPS care map (Additional file 3) provides primary care providers with an algorithm of the summarized CDPS recommendations for primary prevention in 4065 year olds for patients with and without diabetes. A health care provider can use the care map as a decision-making tool during the prevention visit to determine what actions to take when certain conditions are met. This includes consideration as to what CDPS actions a patient is eligible or not eligible to receive, and when to refer a patient back to their primary care provider. For some actions, particularly cardiovascular related CDPS, recommendations depend upon whether a patient has diabetes or not. Use of the CDPS care map is facilitated by information gathered from the patient survey, the prevention visit form and during the prevention visit. Other tools such as the Framingham risk stratification and/or a family history risk assessment tool can also be used to provide further information about the patient's risks of diabetes, cardiovascular disease or cancer.

\section{Bubble diagram}

The bubble diagram (Additional file 5) provides a brief overview of the blended evidence-based CDPS activities for primary prevention in 40-65 year old male and female patients. Regular screening intervals and healthy targets summarized in this tool are meant as a companion piece to the CDPS care map, which depicts the appropriate care path for patients depending on their level of risk. Specific patient details can be entered on a blank version of the bubble diagram and then used as a teaching tool when meeting with patients. The bubble diagrams can facilitate a motivational interviewing approach through agenda setting [39]. For example, after educating the patient about CDPS and while showing the patient their individualized bubble diagram, the clinician can ask the patient what they want to do to improve their health and begin the work that is finalized in the prevention prescription (described below). Intrinsic in the patient-centred approach is the ability of the patient to opt out of discussing any area that they do not wish to address. The bubble diagram allows the negotiation of a shared agenda for the prevention visit through a visual emphasis on the bubbles the patient wants to address.

The bubble diagram with the evidence overview can also be used as a visual cue to remind primary care providers about the CDPS activities to consider when seeing patients in this age group.

\section{Prevention prescription with goals}

The prevention prescription (Additional file 6) includes a summary of the patient's CDPS status, target check-in intervals, referrals or actions to be completed, and any tools provided or linkages made to clinic or community resources to aid the patient in their CDPS efforts. The information on the prevention prescription that does not require shared decision-making can be partially completed before the visit and then finalized with input from the patient at the time of the visit. The goal sheet facilitates shared decision-making through the development of SMART goals including an assessment of confidence addressing action planning and self-efficacy in patient self-management [41, 42]. The prevention prescription with goals can be provided to the patient as a summary of their visit and serve as the patient's personalized CDPS plan.

\section{Discussion}

\section{The need for a BETTER approach to CDPS}

Busy clinicians lack adequate tools and resources to address CDPS in the primary care setting since many guidelines focus on specific conditions [7,9] and lack precise recommendations that are clinically applicable [7]. The BETTER tools bridge the knowledge to action 
gap through a blended approach of actionable items at every step in the process of CDPS from collecting the necessary patient information to care maps for primary care providers and teams [7]. Through engaging the end-users in the process of developing CDPS tools and resources and applying the tools into the clinical setting, the BETTER trial was able to effectively implement CDPS in the family practice setting through a new skilled role of 'prevention practitioner' (PP) [12]. These tools and resources further facilitate knowledge uptake by patients through agenda setting, shared-decisionmaking and self-management. The updated tools and resources described in this paper were refined in order to further facilitate patient assessments, education and shared decision-making aimed to identify and achieve the patient's personalized CDPS goals as well as capture the information required to evaluate CDPS outcomes for the BETTER 2 program implementation [14] in urban, rural, remote and aboriginal settings.

Modifiable lifestyle factors such as smoking, unhealthy eating, physical inactivity and unhealthy alcohol consumption have a huge impact on chronic disease [43] and there is a pressing need to address multiple behavioural risk factors in primary care [44]. Although addressing modifiable lifestyle risk factors can significantly impact mortality and morbidity $[43,45]$ few individuals receive lifestyle counselling, even after a significant illness such as a cardiovascular event $[46,47]$. The updated BETTER 2 tools provide clinicians with resources that evaluate the patient's multiple lifestyle risks including their readiness to change and can be used to track changes over time. In addition, these tools facilitate shared decision-making with patients through agenda setting and identifying specific goals that encourage self-management $[41,42]$.

The tools are tailored to be adaptable and can be used in a number of ways as depicted in Fig. 3. In this way, multidisciplinary teams or family physicians can decide how best to apply the tools in their clinical settings. The updated BETTER approach may be used at the policy and practice level to target at risk populations and invite patients to receive an effective individualized CDPS intervention based on high-level evidence [12] supported by the BETTER 2 tool kit. Moreover, the tools can be harmonized and integrated with existing public health initiatives in various settings. For example, an initiative involving population or practice level CDPS facilitation may consider the BETTER approach or integrating some of the tools to translate population level CDPS activities to individual at-risk patients. Policymakers may provide primary care providers with the BETTER tools to better implement CDPS into practice. Decision makers and primary care providers may adapt the tools to facilitate policy and practice integration of CDPS including consistent messages at all levels.
This approach to knowledge integration is not without its limitations. The tools and resources developed focus on primary prevention of CDPS in patients aged 40-65; hence other high-level interventions such as immunizations, secondary prevention and chronic disease management are not included. The BETTER tools and resources were developed with knowledge integration considered at every step to bridge the research to practice gap through an implementation plan that engaged the end-users and applied the developed resources into the practice setting (Fig. 2). Consequently, the final tools may not reflect the 'best' evidence but the best evidence that could be applied into the settings engaged. Also, over time, the various guidelines change, requiring constant updating and revisions to the tools. Furthermore, the process of knowledge integration is time consuming and requires organization, expertise and resources to conduct. The time and resources required to integrate knowledge into practice settings are not readily available in a health care system that is designed to focus on acute medicine and disease management. In addition to developing clinical practice guidelines, resources should also be used to develop and refine tools and processes, such as the BETTER 2 tools that allow guidelines to be more easily implemented into clinical practice.

The BETTER 2 tools have been implemented and tested in the various practice settings [14] and the outcomes will be presented in a future publication. The BETTER 2 tools can be downloaded for use from the BETTER website [23]. Presently, the tools have been paper based which may limit the ability to implement the PP model into primary care in Canada due to the increased use of electronic medical records in primary care. Electronic versions of the patient survey and prevention prescription are currently being developed and will be tested in primary care settings in Alberta, Newfoundland and Labrador.

Primary care teams should consider implementing the PP role to better address CDPS in conjunction with the primary care provider and thereby share the management of chronic disease prevention and screening. In Canada, prevention and early detection could reduce the burden of managing acute and chronic conditions. Despite that, there are barriers to CDPS. In many jurisdictions, the fee-for-service system does not remunerate prevention activities. Also, some settings lack the clinical resources to address the acute and chronic medical needs of the community. Hence there is limited capacity to implement the PP model in settings that do not compensate for this CDPS or that lack clinical resources to address and manage the acute and chronic conditions. The process could be implemented in other countries with heath care systems that have the resources to support this type of activity. 


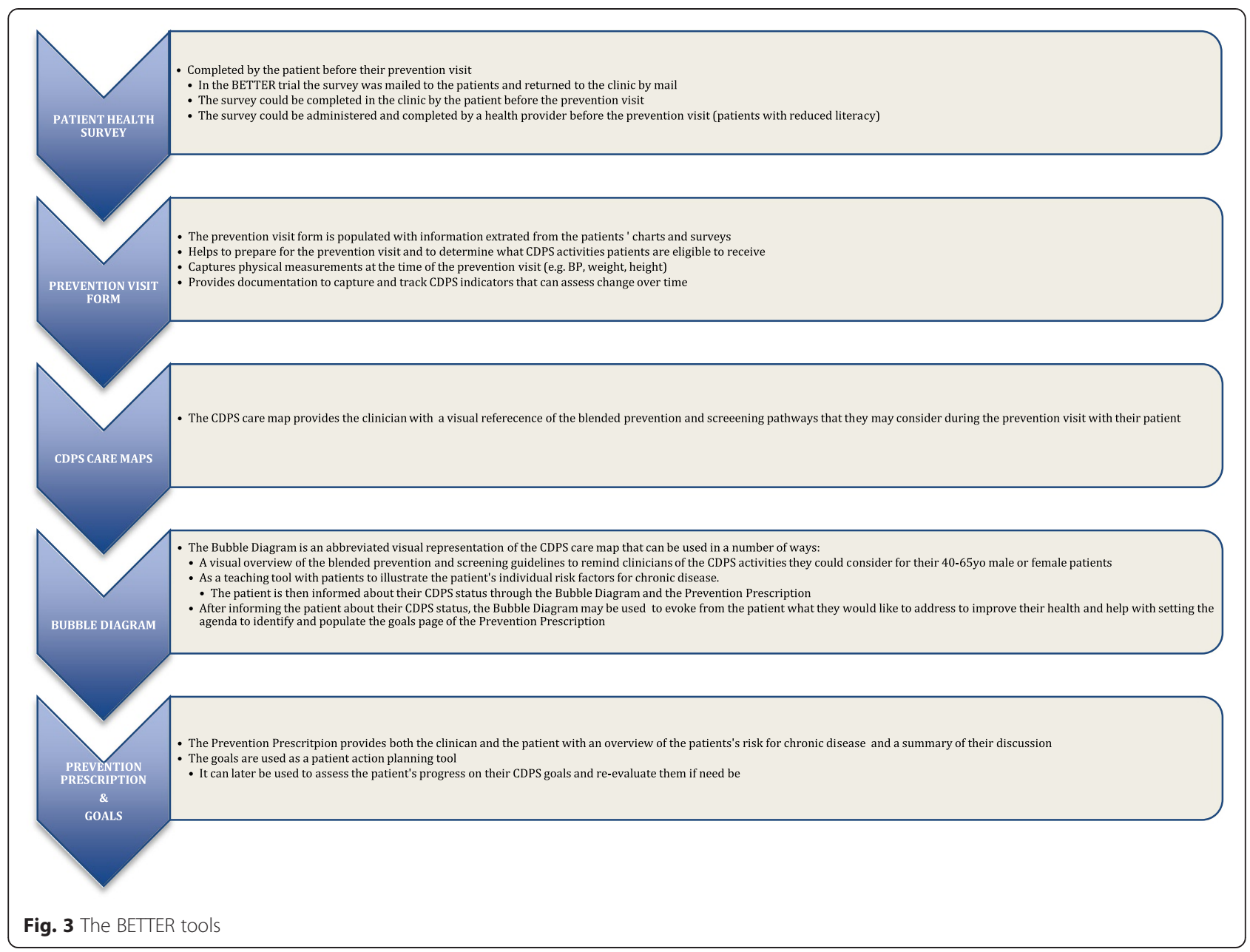

\section{Conclusion}

The BETTER tools are a first step to structure CDPS in primary care in a comprehensive, structured, personalized and evidence-based manner and to improve the application of knowledge into practice. The integrated clinical decision-making tools of BETTER 2 provide a resource for clinicians and policymakers that address patients' complex care needs beyond a single disease approach and can be adapted to facilitate CDPS in various primary care clinical setting in urban, rural and remote communities.

\section{Additional files}

Additional file 1: Patient health survey. (PDF $551 \mathrm{~kb}$ )

Additional file 2: Components and sources of the patient health survey. (DOCX $111 \mathrm{~kb}$ )

Additional file 3: CDPS care maps. (PDF $184 \mathrm{~kb}$ )

Additional file 4: Prevention visit form. (PDF $494 \mathrm{~kb}$ )

Additional file 5: The Bubble diagram. (PDF $934 \mathrm{~kb}$ )

Additional file 6: The prevention prescription. (PDF $354 \mathrm{~kb}$ )

\section{Abbreviations}

BETTER trial: Building on Existing Tools to Improve Chronic Disease

Prevention and Screening in Family Practice; BETTER 2: Building on Existing Tools to Improve Chronic Disease Prevention and Screening in Primary Care; CDPS: chronic disease prevention and screening; CLASP: Coalitions Linking Action and Science for Prevention; CPAC: Canadian Partnership Against Cancer; CWG: clinical working group; PP: prevention practitioner;

SMART: specific, measurable, attainable, realistic, timely.

\section{Competing interests}

The authors declare that they have no competing interests.

\section{Authors' contributions}

We certify that all individuals listed as authors of this manuscript 1) have made substantial contributions to conception and design, or acquisition of data, or analysis and interpretation of data; 2) have been involved in drafting the manuscript or revising it critically for important intellectual content; 3 ) have given final approval of the version to be published; and 4) agree to be accountable for all aspects of the work in ensuring that questions related to the accuracy or integrity of any part of the work are appropriately investigated and resolved. All authors read and approved the final manuscript.

\section{Authors' information}

DPM, DCS, KAB, GS, JC, MG and EG are active family physicians and researchers with content expertise including expertise in qualitative and 
quantitative methodologies. KK is the Deputy Chief Public Health Officer in the Northwest Territories. VF is a nurse and the Territorial Prevention Coordinator for the BETTER 2 program in the Northwest Territories. CA and $\mathrm{JB}$ are research coordinators for the BETTER 2 program. $\mathrm{CM}$ is a biostatistician.

Members of the original BETTER Trial and Clinical Working Group also included the following: R. Barrett, J. Beca, S. Bell, S. Bible, S. Buchman, M. Dhaliwal, S. Dunn, M. Griever, C. Harrison, JS. Hoch, L. Hosford, M. Kahan, D. Klein, P. Krueger, C. Korownyk, K. Lang-Robertson, M Mamdani, J. Meuser, V. Mozgala, L Patterson, J. Pencharz, L. Radmanovich, J. Rogers, L. Schaar, R. Stoller, KE. Thorpe, DL. Vesik, R. Wymer, M. Yan.

\section{Acknowledgements}

Special acknowledgement of Northwest Territory members who assisted with early development of the BETTER 2 tools goes to Jan Inman and Jamie Neudorf. We also would like to acknowledge the BETTER 2 Qualitative Research Lead, Nicolette Sopcak, and Prevention Practitioners: Loriann Lyall, Judy Mercer, Glenda Stagg-Sturge, Margo Wilson.

This research was made possible through a financial contribution from Health Canada through the Canadian Partnership Against Cancer. Dr. Grunfeld is supported by a clinician scientist award from the Ontario Institute for Cancer Research (OICR) with funds from the Ontario Ministry of Research and Innovation (MRI). The opinions, results and conclusions reported herein are those of the authors and do not necessarily reflect the views from the funders. No endorsement by OICR or MRI is intended or should be inferred.

\section{Author details}

1 Department of Family Medicine, University of Alberta, 6-10 University Terrace, Edmonton, Alberta T6G 2T4, Canada. ${ }^{2}$ Covenant Health, Grey Nuns Community Hospital, 1100 Youville Drive Northwest, Edmonton, Alberta T6L $5 \times 8$, Canada. ${ }^{3}$ Discipline of Family Medicine, Memorial University of Newfoundland, 300 Prince Phillip Drive, St. John's, Newfoundland A1B 3V6, Canada. ${ }^{4}$ Department of Health and Social Services, Government of Northwest Territories, P.O. Box 1320, Yellowknife, Northwest Territories X1A 2L9, Canada. ${ }^{5}$ Department of Family and Community Medicine, University of Toronto, 500 University Avenue, Toronto, Ontario M5G 1V7, Canada. 'School of Public Health, University of Alberta, 1140587 Avenue, Edmonton, Alberta T6G 1C9, Canada. ${ }^{7}$ Ontario Institute for Cancer Research, 661 University Avenue, Suite 510, Toronto, Ontario M5G 0A3, Canada.

\section{Received: 1 March 2015 Accepted: 27 July 2015}

\section{Published online: 04 August 2015}

\section{References}

1. Broemeling AM, Watson DE, Prebtani F. Population patterns of chronic health conditions, co-morbidity and healthcare use in Canada: implications for policy and practice. Healthc Q. 2008;11(3):70-6.

2. Geneau R, Stuckler D, Stachenko S, McKee M, Ebrahim S, Basu S, et al. Raising the priority of preventing chronic diseases: a political process. Lancet. 2010;376(9753):1689-98.

3. Mclsaac W, Fuller-Thomson E, Talbot Y. Does having regular care by a family physician improve preventive care? Can Fam Physician. 2001;47:70-6.

4. Upshur R, Leong A, Maaten S, Wang L. Patterns of primary and secondary prevention. In: Jaakkimainen L, Upshur R, Klein-Geltink J, et al., editors. Primary Care in Ontario: ICES Atlas. Toronto: Institute for Clinical and Evaluative Sciences; 2006. p. 78-89.

5. Starfield B, Shi L, Macinko J. Contribution of primary care to health systems and health. Milbank Q. 2005;83(3):457-502.

6. Grol R. Successes and failures in the implementation of evidence-based guidelines for clinical practice. Med Care. 2001;39(8 Suppl 2):I146-54.

7. Campbell-Scherer D, Rogers J, Manca D, Lang-Robertson K, Bell S, Salvalaggio G, et al. Guideline harmonization and implementation plan for the BETTER trial: building on Existing Tools to Improve Chronic Disease Prevention and Screening in Family Practice. CMAJ Open. 2014;2(1):E1-E10.

8. Hoffman C, Rice D, Sung HY. Persons with chronic conditions. Their prevalence and costs. JAMA. 1996;276(18):1473-9.

9. Tobe SW, Stone JA, Brouwers M, Bhattacharyya O, Walker KM, Dawes M, et al. Harmonization of guidelines for the prevention and treatment of cardiovascular disease: the C-CHANGE Initiative. CMAJ. 2011;183(15):E1135-1150.
10. Graham ID, Logan J, Harrison MB, Straus SE, Tetroe J, Caswell W, et al. Lost in knowledge translation: time for a map? J Contin Educ Health Prof. 2006;26(1):13-24

11. Yarnall KS, Pollak Kl, Ostbye T, Krause KM, Michener JL. Primary care: is there enough time for prevention? Am J Public Health. 2003;93(4):635-41.

12. Grunfeld E, Manca D, Moineddin R, Thorpe KE, Hoch JS, Campbell-Scherer $D$, et al. Improving chronic disease prevention and screening in primary care: results of the BETTER pragmatic cluster randomized controlled trial. BMC Fam Pract. 2013;14(1):175.

13. Manca DP, Greiver M, Carroll JC, Salvalaggio G, Cave A, Rogers J, et al. Finding a BETTER way: a qualitative study exploring the prevention practitioner intervention to improve chronic disease prevention and screening in family practice. BMC Fam Pract. 2014;15(1):66.

14. Manca DP, Aubrey-Bassler K, Kandola K, Aguilar C, Campbell-Scherer D, Sopcak $N$, et al. Implementing and evaluating a program to facilitate chronic disease prevention and screening in primary care: a mixed methods program evaluation. Implement Sci. 2014;9:135.

15. Cargo M, Mercer SL. The value and challenges of participatory research: strengthening its practice. Annu Rev Public Health. 2008;29:325-50.

16. Bowen S, Martens PJ. A model for collaborative evaluation of universitycommunity partnerships. J Epidemiol Community Health. 2006;60(10):902-7.

17. Golden-Biddle K, Reay T, Petz S, Witt C, Casebeer A, Pablo A, et al. Toward a communicative perspective of collaborating in research: the case of the researcher-decision-maker partnership. J Health Serv Res Policy. 2003:8 Suppl 2:20-5.

18. Lomas J. Using 'linkage and exchange' to move research into policy at a Canadian foundation. Health Aff (Millwood). 2000;19(3):236-40.

19. Butt P, Canadian Centre on Substance Abuse. Alcohol and health in Canada a summary of evidence and guidelines for low-risk drinking. Ottawa, Ont: Canadian Centre on Sustance Abuse; 2011. p. 1. electronic text (66 p.).

20. Fedirko V, Tramacere I, Bagnardi V, Rota M, Scotti L, Islami F, et al. Alcohol drinking and colorectal cancer risk: an overall and dose-response metaanalysis of published studies. Ann Oncol. 2011;22(9):1958-72.

21. Seitz HK, Pelucchi C, Bagnardi V, La Vecchia C. Epidemiology and pathophysiology of alcohol and breast cancer: update 2012. Alcohol Alcohol. 2012;47(3):204-12.

22. Canadian Cancer Society. Prevention \& Screening: Living Well. Alcohol. 2014 [http://www.cancer.ca/en/prevention-and-screening/live-well/alcohol/? region $=$ on]

23. The BETTER Program. [http://better.utoronto.ca/]

24. Blair SN, Haskell WL, Ho P, Paffenbarger Jr RS, Vranizan KM, Farquhar JW, et al. Assessment of habitual physical activity by a seven-day recall in a community survey and controlled experiments. Am J Epidemiol. 1985;122(5):794-804.

25. Kris-Etherton P, Eissenstat B, Jaax S, Srinath U, Scott L, Rader J, et al. Validation for MEDFICTS, a dietary assessment instrument for evaluating adherence to total and saturated fat recommendations of the National Cholesterol Education Program Step 1 and Step 2 diets. J Am Diet Assoc. 2001;101(1):81-6.

26. Taylor AJ, Wong H, Wish K, Carrow J, Bell D, Bindeman J, et al. Validation of the MEDFICTS dietary questionnaire: a clinical tool to assess adherence to American Heart Association dietary fat intake guidelines. Nutr J. 2003;2:4.

27. Heron N, Tully MA, McKinley MC, Cupples ME. Physical activity assessment in practice: a mixed methods study of GPPAQ use in primary care. BMC Fam Pract. 2014;15:11.

28. Kirkman S, Dunbar SA. American Diabetes Association Clinical Practice Recommendations 2010 Introduction. Diabetes Care. 2010;33:S1-2.

29. Scottish Intercollegiate Guidelines Network. Risk estimation and the prevention of cardiovascular disease: a national clinical guideline. Edinburgh, Scotland: SIGN; 2007.

30. Colberg SR, Sigal RJ, Fernhall B, Regensteiner JG, Blissmer BJ, Rubin RR, et al. Exercise and type 2 diabetes: the American College of Sports Medicine and the American Diabetes Association: joint position statement. Diabetes Care. 2010;33(12):e147-167.

31. Riethof $M$, Flavin PL, Lindvall B, Michels $R, O^{\prime}$ Connor $P$, Redmon $B$, et al. Health care guideline : diagnosis and management of type 2 diabetes mellitus in adults. 15th ed. Bloomington, Minn: ICSI; 2012.

32. American Diabetes A. Standards of medical care in diabetes-2012. Diabetes Care. 2012;35 Suppl 1:S11-63.

33. Paxton AE, Strycker LA, Toobert DJ, Ammerman AS, Glasgow RE. Starting the conversation performance of a brief dietary assessment and intervention tool for health professionals. Am J Prev Med. 2011;40(1):67-71. 
34. National Institute on Alcohol Abuse and Alcoholism. Alcohol \& Health. Overview of Alcohol Consumption. Moderate \& Binge Drinking [http://www.niaaa.nih.gov/alcohol-health/overview-alcohol-consumption/ moderate-binge-drinking]

35. Bush K, Kivlahan DR, McDonell MB, Fihn SD, Bradley KA. The AUDIT alcohol consumption questions (AUDIT-C): an effective brief screening test for problem drinking. Ambulatory Care Quality Improvement Project (ACQUIP). Alcohol Use Disorders Identification Test. Arch Intern Med. 1998;158(16):1789-95.

36. Alcohol Screening, Brief Intervention \& Referral: helping patients reduce alcohol-related risks [http://www.sbir-diba.ca/]

37. Problem Drinking. [http://www2.gov.bc.ca/gov/content/health/ practitionerprofessional-resources/bc-guidelines/problem-drinking]

38. Bradley KA, Bush KR, Epler AJ, Dobie DJ, Davis TM, Sporleder JL, et al. Two brief alcohol-screening tests From the Alcohol Use Disorders Identification Test (AUDIT): validation in a female Veterans Affairs patient population. Arch Intern Med. 2003;163(7):821-9.

39. Rollnick S, Miller WR, Butler C. Motivational interviewing in health care: helping patients change behavior. New York: Guilford Press; 2008

40. Lorig KR, Sobel DS, Ritter PL, Laurent D, Hobbs M. Effect of a selfmanagement program on patients with chronic disease. Eff Clin Pract. 2001;4(6):256-62.

41. Bodenheimer T, Lorig K, Holman H, Grumbach K. Patient self-management of chronic disease in primary care. JAMA. 2002;288(19):2469-75.

42. Lorig KR, Holman H. Self-management education: history, definition, outcomes, and mechanisms. Ann Behav Med. 2003:26(1):1-7.

43. Haydon E, Ontario Chronic Disease Prevention Alliance, Ontario Public Health Association. Chronic disease in Ontario and Canada determinants, risk factors and prevention priorities. Toronto, Ont: Ontario Chronic Disease Prevention Alliance; 2006. p. 1. electronic text (x, 212 p.).

44. Pronk NP, Peek CJ, Goldstein MG. Addressing multiple behavioral risk factors in primary care. A synthesis of current knowledge and stakeholder dialogue sessions. Am J Prev Med. 2004;27(2 Suppl):4-17.

45. Lee IM, Shiroma EJ, Lobelo F, Puska P, Blair SN, Katzmarzyk PT, et al. Effect of physical inactivity on major non-communicable diseases worldwide: an analysis of burden of disease and life expectancy. Lancet. 2012;380(9838):219-29.

46. Roblin D, Diseker 3rd RA, Orenstein D, Wilder M, Eley M. Delivery of outpatient cardiac rehabilitation in a managed care organization. J Cardpulm Rehabil. 2004:24(3):157-64.

47. Suaya JA, Shepard DS, Normand SL, Ades PA, Prottas J, Stason WB. Use of cardiac rehabilitation by Medicare beneficiaries after myocardial infarction or coronary bypass surgery. Circulation. 2007;116(15):1653-62.

\section{Submit your next manuscript to BioMed Central and take full advantage of:}

- Convenient online submission

- Thorough peer review

- No space constraints or color figure charges

- Immediate publication on acceptance

- Inclusion in PubMed, CAS, Scopus and Google Scholar

- Research which is freely available for redistribution

Submit your manuscript at www.biomedcentral.com/submit 